\title{
Intra-Accumbens Amphetamine Increases the Conditioned Incentive Salience of Sucrose Reward: Enhancement of Reward "Wanting" without Enhanced "Liking" or Response Reinforcement
}

\author{
Cindy L. Wyvell and Kent C. Berridge \\ Department of Psychology, University of Michigan, Ann Arbor, Michigan 48109
}

\begin{abstract}
Amphetamine microinjection into the nucleus accumbens shell enhanced the ability of a Pavlovian reward cue to trigger increased instrumental performance for sucrose reward in a pure conditioned incentive paradigm. Rats were first trained to press one of two levers to obtain sucrose pellets. They were separately conditioned to associate a Pavlovian cue (30 sec light) with free sucrose pellets. On test days, the rats received bilateral microinjection of intra-accumbens vehicle or amphetamine $(0.0,2.0$, 10.0 , or $20.0 \mu \mathrm{g} / 0.5 \mu \mathrm{l})$, and lever pressing was tested in the absence of any reinforcement contingency, while the Pavlovian cue alone was freely presented at intervals throughout the session. Amphetamine microinjection selectively potentiated the cue-elicited increase in sucrose-associated lever pressing, although instrumental responding was not reinforced by either sucrose or the cue during the test. Intra-accumbens amphet-
\end{abstract}

amine can therefore potentiate cue-triggered incentive motivation for reward in the absence of primary or secondary reinforcement. Using the taste reactivity measure of hedonic impact, it was shown that intra-accumbens amphetamine failed to increase positive hedonic reaction patterns elicited by sucrose (i.e., sucrose "liking") at doses that effectively increase sucrose "wanting." We conclude that nucleus accumbens dopamine specifically mediates the ability of reward cues to trigger "wanting" (incentive salience) for their associated rewards, independent of both hedonic impact and response reinforcement.

Key words: dopamine; hedonia; instrumental; learning; palatability; motivation, ventral striatum; mesolimbic; limbic; shell; addiction; taste reactivity; reward; conditioned stimulus; incentive; reinforcement; Pavlovian; incentive salience
Dopaminergic activity in the nucleus accumbens is implicated in motivation for food and other incentives (Hernandez and Hoebel, 1988; Blackburn et al., 1989; Phillips et al., 1993; Mark et al., 1994; Richardson and Gratton, 1996; Di Ciano et al., 1998a,b; Ahn and Phillips, 1999), and intra-accumbens microinjection of dopamine (DA) agonists can increase food intake and instrumental performance for food-related cues (Cador et al., 1991; Burns et al., 1993; Sills and Vaccarino, 1996; Swanson et al., 1997). However, the specific role of dopamine in reward remains debated.

The incentive salience hypothesis of dopamine function in reward suggests that accumbens dopamine neurotransmission modulates the attribution of incentive salience to reward cues, which triggers a motivational state of "wanting" for both the cue and its associated reward (Berridge and Valenstein, 1991; Robinson and Berridge, 1993; Berridge and Robinson, 1998). As alternative explanations for the role of dopamine in reward, the hedonia hypothesis suggests that dopamine mediates the hedonic impact of rewards (Wise et al., 1978; Wise, 1985; Gardner and Lowinson, 1993; Volkow et al., 1999). Reward learning hypotheses suggest that dopamine mediates either response reinforcement or associative predictions of reward (Di Chiara, 1998; McFarland and Ettenberg, 1998; Schultz et al., 1998), and there are still other conceptual alternatives, too (Salamone et al., 1997; Sokolowski and Salamone, 1998; Everitt et al., 2000).

It is clear that dopamine modulates the effects of Pavlovian reward cues on motivated behavior. For example, microinjection of intra-accumbens amphetamine potentiates instrumental responding that is reinforced by a food cue (Taylor and Robbins, 1984;

Received March 27, 2000; revised July 24, 2000; accepted July 27, 2000.

This research was supported by National Science Foundation Grant IBN 9604408 (to K.C.B.) and National Institutes of Health National Research Service Award Fellowship F31 DA0599901 (to C.L.W.). We thank Profs. J. Wayne Aldridge, Anthony Dickinson, Barry Everitt, and Terry Robinson, and Drs. John Parkinson and Jeremy Hall, for helpful comments on an earlier version of this manuscript.

Correspondence should be addressed to Cindy Wyvell or Kent Berridge, Department of Psychology, University of Michigan, Ann Arbor, MI 48109-1109. E-mail: wyvell@umich.edu; berridge@umich.edu.

Copyright (C) 2000 Society for Neuroscience $0270-6474 / 00 / 208122-09 \$ 15.00 / 0$
Robbins et al., 1989; Kelley and Delfs, 1991), which has led some investigators to suggest that accumbens dopamine modulates control of behavior by secondary reinforcers (Everitt and Robbins, 1992; Phillips et al., 1994; Everitt et al., 2000). However, it is unclear from secondary reinforcement paradigms whether the effects of accumbens dopamine actually require response reinforcement by the cue or instead can be mediated purely by the incentive motivational properties of the cue.

A pure conditioned incentive paradigm more selectively measures the role of dopamine in motivational processes such as incentive salience, because it measures the ability of a cue to trigger motivation for rewards in the absence of both primary and secondary reinforcement (Rescorla and Solomon, 1967; Toates, 1986; Dickinson and Dawson, 1987; Balleine, 1994; Dickinson and Balleine, 1994). Primary reinforcement is avoided in a conditioned incentive paradigm by performing the test under extinction conditions, so that no food is actually earned by responding. Secondary reinforcement is also avoided by not reinforcing the instrumental response with the food cue. The cue is instead presented freely at intervals throughout the session, which restricts it to eliciting behavior rather than reinforcing behavior. According to the incentive salience hypothesis, a reward cue is attributed with incentive salience by dopamine-related neural systems, causing the cue to trigger "wanting" and to potentiate instrumental performance for that reward. The conditioned incentive paradigm therefore provides a relatively specific way to test the hypothesis that intraaccumbens amphetamine increases incentive salience attribution without being confounded by response reinforcement processes. A role for dopamine in conditioned incentive motivation has been indicated recently by Dickinson et al. (2000). They used a conditioned incentive paradigm to show that systemic dopamine antagonists specifically blocked the ability of a Pavlovian reward cue to trigger increases in responding for the associated reward (Dickinson et al., 2000).

The purpose of our study was to investigate the role of accumbens dopamine in incentive salience attribution. Microinjections of the indirect dopamine agonist amphetamine were administered 
into the accumbens shell to enhance dopamine neurotransmission. The first experiment investigated whether intra-accumbens amphetamine potentiates cue-triggered "wanting" for sucrose by enhancing the incentive salience of the cue, using the conditioned incentive paradigm. A related experiment investigated whether doses of intra-accumbens amphetamine that increased "wanting" for sucrose would also increase "liking" for sucrose, using the affective taste reactivity technique to measure hedonic impact (Grill and Norgren, 1978; Berridge, 2000a).

\section{MATERIALS AND METHODS}

\section{Experiment 1: instrumental testing}

Subjects. Thirty female Sprague Dawley rats (born at the University of Michigan) weighing between 220 and $330 \mathrm{gm}$ were housed in pairs under a reverse $12 \mathrm{hr}$ light/dark cycle (lights off at 9:00 A.M.). Rats were kept in plastic tub cages in a temperature- and humidity-controlled room. Subjects had access to water ad libitum and were given $20-25 \mathrm{gm}$ of rat chow each day after the training or test session. This feeding regimen was designed to maintain the subjects on a regular feeding schedule without significantly depriving them of food and yet keep them motivated to work for food reward. A pure conditioned incentive paradigm was used, which followed the Pavlovian instrumental transfer procedures for training and behavioral testing developed by Dickinson and colleagues (Dickinson and Dawson, 1987; Dickinson and Balleine, 1994; Dickinson et al., 2000).

Instrumental training. The four operant chambers (Med Associates Inc., St. Albans, VT) were made of stainless steel and Plexiglas and contained a house light, sucrose cup, and two levers. For the primary experimental groups, a small cue light bulb, embedded inside a Plexiglas block $(4.5 \times$ $2.5 \times 1.0 \mathrm{~cm}$ ), was mounted onto the top of each lever. This allowed the cue lights to appear to be placed within the levers themselves to facilitate Pavlovian influence over instrumental lever pressing (Tomie, 1996). The operant boxes were enclosed in sound attenuating chambers and controlled by Med Associates Inc. interface equipment and a personal computer.

Rats were first given two sessions of magazine training to shape them to eat from the sucrose cup. In these sessions, $45 \mathrm{mg}$ sucrose pellets (Formula F; P. J. Noyes Co., Lancaster, PA) were delivered on a fixed-time, $1 \mathrm{~min}$ schedule of reinforcement. Next, across 14 consecutive days, the rats were trained to lever press for sucrose pellets during daily $30 \mathrm{~min}$ sessions, and the schedule of reinforcement was gradually incremented up to a variableinterval, $45 \mathrm{sec}$ schedule. One lever in the chamber was designated as the sucrose lever, and presses on it produced sucrose pellets. Responses on the other lever (nonsucrose control lever) produced no consequence and were used to assess changes in general sensorimotor arousal produced by amphetamine during the test sessions.

Pavlovian training. After instrumental training, the primary experimental rats were randomly divided into two groups to receive either contingent [conditioned stimulus group (CS + group)] $(n=10)$ or noncontingent (CS random group) $(n=10)$ training with a visual cue in the operant chambers. During Pavlovian conditioning, the levers were left inside the chambers but rendered immobile so that the subjects could not lever press. In each of five sessions, the cue light located on the previously designated sucrose lever was illuminated (CS) 10 times for a $30 \mathrm{sec}$ duration on a variable-time 3-min schedule. For the $\mathrm{CS}+$ group, three sucrose pellets [unconditioned stimulus (UCS)] were delivered into the sucrose cup immediately after the offset of the CS. Thus, a total of 50 CS-UCS pairings were given during the Pavlovian training sessions. The control $\mathrm{CS}_{\mathrm{randon}}$ group also received 50 presentations of the CS and 50 presentations of the UCS, but their temporal correlation with each other was random, because they were presented on two different variable-time, 3 min schedules. The $\mathrm{CS}_{\text {random }}$, therefore, served as a truly random control stimulus (i.e., there was no predictive relationship between light and sucrose for the control group) rather than a negative predictor or inhibitory stimulus (Rescorla, 1967).

Nonlocalized CS control group. A separate group of rats was used to ascertain that the ability of the reward cue to trigger increases in instrumental responding for reward was not simply mediated by Pavlovian approach responses and did not depend on the location of the CS+ physical stimulus. A localized CS + (i.e., cue light embedded within the lever) was used for the primary experimental groups, because discrete localized cues may be best at triggering incentive salience attribution and best at driving cue-triggered goal-directed behavior (Tomie, 1996). However, it is also crucial to show that amphetamine alters the ability of a CS+ to increase instrumental behavior by increasing the incentive value of the sucrose reward itself and not merely conditioned approach behavior to the Pavlovian CS +. Finally, it was deemed useful to have a within-subject comparison of CS + versus CS - effects in this paradigm, as well.

For these reasons, the nonlocalized control group $(n=10)$ received Pavlovian training with two auditory stimuli (tone and clicker) as CS + and CS-, which were presented from wall-mounted speakers. The CSs were therefore not localized to the sucrose lever, control lever, sucrose dish, or any other reward-relevant object in the chamber. Additionally, both levers were removed entirely from the operant chambers during Pavlovian conditioning to further prevent the formation of any possible Pavlovian association between a lever and the sucrose UCS. Across each of 14 sessions, the CS+ (either a $30 \mathrm{sec}$ pulsing $2.9 \mathrm{kHz}$ tone or clicker; stimuli balanced across rats) was presented 10 times on a variable-time, $1.5 \mathrm{~min}$ schedule and contingently followed by the UCS (three sucrose pellets). The CS- (the other auditory stimulus) was also presented once in the middle and once at the end of the last three Pavlovian training sessions and was never followed by sucrose pellets.

Surgery. After the completion of Pavlovian training, all rats were anesthetized with ketamine $(80 \mathrm{mg} / \mathrm{kg})$ and Rompun $(5 \mathrm{mg} / \mathrm{kg})$ and were stereotaxically implanted with bilateral 22 gauge cannulas targeted at the shell of the nucleus accumbens. A slanted skull position was used, with the incisor bar set at $+5.0 \mathrm{~mm}$, and the coordinates were $3.1 \mathrm{~mm}$ anterior to bregma, $1.0 \mathrm{~mm}$ lateral to the midline, and $5.7 \mathrm{~mm}$ ventral to the skull surface. The cannulas were anchored with skull screws and cranial cement, and stylets were used to prevent cannula occlusion. The rats were given postoperative penicillin and were allowed to recover from surgery for 1 week before the actual test sessions began.

Drugs and microinjections. D-Amphetamine sulfate (Sigma, St. Louis, MO) was dissolved in sterile $0.9 \%$ saline $(0.0,2.0,10.0$, or $20.0 \mu \mathrm{g} / 0.5 \mu \mathrm{l}$; dose refers to amount given per side, and microinjections were always given bilaterally). The testing order of drug doses and vehicle was counterbalanced between subjects using a $4 \times 4$ Latin square design. Microinjections were conducted with an infusion pump while the rats were gently hand-held. The microinjector tips ( 28 gauge) extended $2.5 \mathrm{~mm}$ beyond the guide cannulas, and the drug injections were performed over a period of 1 min, with the microinjectors left in place for an additional $1 \mathrm{~min}$ period to allow for drug diffusion. Before testing, one vehicle injection was conducted to minimize the mechanical effects of subsequent microinjections and to habituate the rats to the injection procedure. The rats were then returned to their home cages.

Testing effect of intra-accumbens amphetamine and cue presentations on lever pressing. A few days after surgery, all rats were given three additional training sessions of lever pressing on a variable-interval, $45 \mathrm{sec}$ schedule with sucrose pellet reinforcement to reestablish instrumental performance. These sessions were followed by one extinction session of lever pressing to expose the rats to extinction conditions, and then the actual testing began.

During each of four 30 min test sessions, the rats in the primary experimental groups were first given bilateral microinjections of vehicle or amphetamine (order of $0.0,2.0,10.0$, or $20.0 \mu \mathrm{g}$ doses was counterbalanced across subjects), and then they were placed into the operant chambers. Instrumental performance was assessed under extinction conditions (i.e., no sucrose pellets were given during the test, so there was no primary reinforcement of pressing responses; similarly, the cue was no longer followed by sucrose, so there was no Pavlovian reinforcement; finally, pressing was not rewarded by the cue light, so there was no secondary reinforcement of responses). In each test session, five free $30 \mathrm{sec}$ cue presentations were given on a fixed-time, 4 min schedule (for the nonlocalized CS control group, the $30 \mathrm{sec}$ tone and clicker auditory stimuli were each presented four times in alternating order; each rat was tested in two sessions after microinjection of either vehicle or $5.0 \mu \mathrm{g}$ amphetamine in counter-balanced order). Responding on the sucrose and nonsucrose control levers was recorded throughout the session. The number of lever presses that occurred during each $30 \mathrm{sec}$ cue presentation and the number of lever presses that occurred during the $30 \mathrm{sec}$ period immediately before each cue presentation were each marked for special analysis to compare responding triggered by the cue to responding in the absence of the cue. Test sessions were spaced every $48 \mathrm{hr}$.

Histology. After the completion of testing, the rats were deeply anesthetized with sodium pentobarbital and transcardially perfused with saline and formaldehyde. The brains were extracted, sliced coronally into $50 \mu \mathrm{m}$ sections, and stained with cresyl violet. Cannula placements were verified, and six subjects with cannula placements located outside of the nucleus accumbens shell were excluded from the data analysis, which resulted in a final total of eight subjects per group.

Statistics. Because the variance of the data were found to increase with the mean, total scores of lever pressing during the responding bouts were first square root transformed to achieve homogeneity of variance as assessed by the Mauchly Sphericity test. ANOVAs were then performed to examine the between-subject variable of group $\left(\mathrm{CS}+\mathrm{vs} \mathrm{CS}_{\text {random }}\right)$ and the within-subject variables of drug (vehicle or dose, $0,2,10$, or $20 \mu \mathrm{g}$ ), cue (CS light illumination vs absence of cue light), and lever (sucrose lever vs nonsucrose control lever). When main effects and interactions were found, the Bonferroni method was used for post hoc comparisons (Glantz, 1997).

\section{Experiment 2: taste reactivity testing}

Measurement of hedonic impact: the taste reactivity paradigm. The taste reactivity paradigm (Grill and Norgren, 1978) provides a way of specifically assessing the hedonic impact of a sucrose reward by measuring affective reactions elicited by oral infusions of taste stimuli from human infants, apes, monkeys, or rats (Grill and Berridge, 1985; Berridge, 1996, 2000a) (see Fig. 5). Positive hedonic reaction patterns (involving rhythmic tongue protrusion, etc.) are normally elicited by sucrose. Negative aversive reaction patterns (involving gapes, etc.) are normally elicited by quinine. Positive hedonic taste reactivity patterns of rats are increased by many of the same physiological, psychological, and pharmacological manipulations that increase human subjective ratings of taste palatability (cf. Berridge, 2000a). For example, hedonic reaction patterns of rats to tastes are enhanced by physiological states such as food deprivation or sodium deple- 
tion (Berridge et al., 1984; Berridge, 1991), similar to the way human subjective ratings of taste palatability are increased by those states $(\mathrm{Ca}$ banac, 1979; Beauchamp et al., 1990; Laeng et al., 1993). Conditioned food preferences based on associative learning also increase positive hedonic taste reactivity patterns, because conditioned stimuli that have been paired with palatable tastes can acquire the ability to enhance hedonic taste reactions (Delamater et al., 1986; Berridge and Schulkin, 1989; Breslin et al., 1990).

Pharmacological manipulations that enhance the hedonic impact of a taste in the taste reactivity paradigm produce selective increases in positive hedonic reaction patterns and either no change or a reduction in negative aversive reaction patterns. For instance, systemic and intracranial injections of opioid agonists or of benzodiazepines have been shown to enhance hedonic palatability using the taste reactivity technique (Treit and Berridge, 1990; Doyle et al., 1993; Peciña and Berridge 1996, 2000). Most relevant to this study, Peciña and Berridge (2000) found that microinjections of an opioid agonist into the accumbens shell increased food intake and enhanced hedonic reaction patterns to an oral infusion of sucrose (but did not increase aversive reactions to quinine). Thus, opioid receptor activation in accumbens shell selectively potentiated the positive hedonic impact of a taste, apparently making food more "liked," as well as more "wanted." In the current study, the amphetamine microinjection sites that increased the conditioned motivation for sucrose reward were clustered around the same accumbens shell site in which morphine microinjection enhanced the hedonic impact of sucrose (Peciña and Berridge, 2000). If amphetamine microinjection in the accumbens increases sucrose "liking" in addition to sucrose "wanting," as morphine microinjection does, then amphetamine microinjection in this site ought to also enhance hedonic taste reactivity patterns elicited by sucrose (and similarly fail to enhance aversive reactions to quinine). Conversely, if amphetamine increases "wanting" but not "liking," as the incentive salience hypothesis of dopamine function predicts, then hedonic reaction patterns should not be altered by amphetamine at this site.

Subjects. Fourteen female Sprague Dawley rats (born at the University of Michigan) weighing between 215 and $315 \mathrm{gm}$ were housed in pairs under a reverse $12 \mathrm{hr}$ light/dark cycle (lights off at 9:00 A.M.). Rats were kept in plastic tub cages, and access to water and rat chow was provided ad libitum.

Surgery. Rats were implanted with bilateral 22 gauge microinjection cannula targeted at the shell of the nucleus accumbens. The stereotaxic coordinates were the same as those used in the first experiment: anteroposterior, $+3.1 \mathrm{~mm}$; lateromedial, $+1.0 \mathrm{~mm}$; and dorsoventral, $-5.7 \mathrm{~mm}$, with the incisor bar set at $+5.0 \mathrm{~mm}$. Rats were also implanted with chronic oral cannulas to enable the infusion of a taste solution into the mouth. A 19 gauge needle connected to cannula tubing was inserted adjacent to the first auxiliary molar and routed along the zygomatic arch to exit at an incision at the scalp. The bilateral oral cannulas (heat-flared polyethylene100 tubing) were anchored inside the mouth by Teflon washers and fitted with 19 gauge cannulas at the skull. Cranial cement was applied to skull screws to secure the brain microinjection cannulas and oral cannulas. Rats were given postoperative penicillin and were allowed to recover from surgery for 1 week before testing.

Taste reactivity testing. Rats were first habituated to the taste reactivity procedure with oral infusions of distilled water. They also received one habituation session in which they received an oral infusion of the solution used for taste reactivity testing. This bittersweet solution was $0.1 \mathrm{M}$ sucrose and $1.5 \times 10^{-4} \mathrm{M}$ quinine hydrochloride in simultaneous concentration. The sucrose-quinine mixture was chosen to elicit both hedonic and aversive affective reaction patterns, which would allow assessment of whether intra-accumbens amphetamine selectively increased positive hedonic reactions.

Drug microinjections were performed using the identical procedures described in the first experiment. Immediately after a drug or vehicle microinjection $(0.0,2.0,10.0$, or $20.0 \mu \mathrm{g} / 0.5 \mu \mathrm{l}$, bilaterally; vehicle and dose order was counter-balanced between subjects), the stimulus delivery tube from the sucrose-quinine syringe was inserted into one of the oral cannula, and then the rat was placed into a plastic chamber with a transparen floor. Ten minutes later, a $1 \mathrm{ml}$ infusion of the taste solution was delivered by an infusion pump over a $1 \mathrm{~min}$ period. The rat's behavior was videotaped with two stationary cameras via a mirror that was mounted beneath the chamber floor. The $1 \mathrm{ml}$ taste infusion was repeated again $10 \mathrm{~min}$ after the completion of the first taste infusion.

The four test sessions were each separated by $48 \mathrm{hr}$ and, as conducted in the first experiment, histology was performed to verify microinjection cannula placement. Three subjects were subsequently found to have microinjection cannula placements located outside of the nucleus accumbens shell and so were excluded from the data analysis to yield a final total of 11 subjects.

Taste reactivity scoring. The videotapes were scored in slow motion (1/10 of actual speed) by an observer blind to drug treatment condition. Both tapes (one from each camera) were scored simultaneously. The use of two stationary cameras ensured a close-up view of the rats' faces and forelimbs while eliminating off-camera time. Hedonic reactions included lateral tongue protrusions (large protrusions of the tongue extending away from the midline), midline tongue protrusions (smaller rhythmic midline protrusions), and paw licking. Aversive reactions included gapes (large opening of the mouth with a retraction of the lower lip), forelimb flails (rapid waving of one or both forelimbs), head shakes, face wiping, and chin rubs

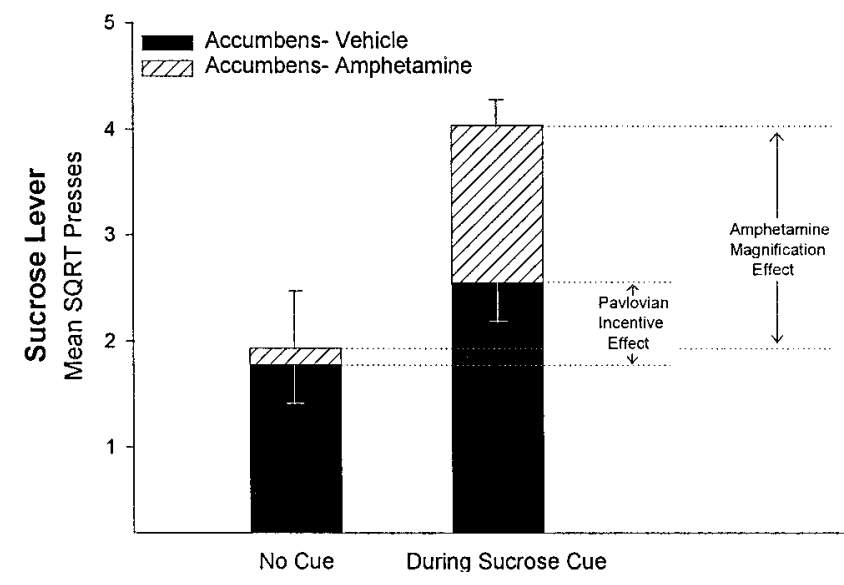

Figure 1. The effect of $\mathrm{CS}+$ cue presentations and amphetamine microinjection (20 $\mu \mathrm{g}$ dose depicted) on responding for sucrose during extinction conditions. Presentation of the Pavlovian sucrose cue ordinarily triggered a moderate increase in responding on the sucrose-associated lever. Intraaccumbens amphetamine nearly tripled the incentive impact triggered by the $\mathrm{CS}+$ sucrose cue, expressed by the increase in pressing elicited by the cue, but amphetamine had relatively little effect on pressing in the absence of the cue.

(pushing chin against the side or floor of the test chamber). Scoring followed the procedures described by Berridge and Peciña (1995). Discrete actions were counted each time they occurred and included lateral tongue protrusions, gapes, forelimb flails, head shakes, and chin rubs. Continuous actions were counted in time bins and included midline tongue protrusions ( 2 sec bins), paw licking ( $5 \mathrm{sec}$ bins), and face wiping ( $5 \mathrm{sec}$ bins).

Statistics. Total hedonic or aversive reactions, as well as individual behavioral components within each affective grouping, were analyzed with one-way repeated measures Friedman ANOVAs (main factor of drug dose). When statistical significance was reached, the Tukey method was used for post hoc comparisons (Glantz, 1997).

\section{RESULTS}

\section{Experiment 1: instrumental testing}

An initial four-way mixed ANOVA was performed for the factors group, drug, lever, and cue. All rats pressed more often on the sucrose lever than on the nonsucrose control lever during the extinction tests $\left(F_{(1,270)}=74.246 ; p<0.001\right)$. There were also significant effects on lever pressing for the drug dose factor $\left(F_{(3,270)}\right.$ $=3.164 ; p<0.05)$ and the Pavlovian cue presence $\left(F_{(1,270)}=\right.$ 30.651; $p<0.001)$, and there was a significant interaction between cue $\times$ group $\left(F_{(1,270)}=5.671 ; p<0.05\right)$. The specific effects of cue presentations and amphetamine microinjections were further analyzed in the $\mathrm{CS}+$ and $\mathrm{CS}_{\text {random }}$ groups as described below.

\section{Effect of cue presentations on lever pressing}

Presentation of the CS + sucrose cue significantly increased lever pressing compared with responding in the absence of the cue (three-way within-subject ANOVA with cue, drug, and lever as factors (main effect of cue, $F_{(1,127)}=23.46 ; p<0.005$ ) (Fig. 1), but the effect of the CS+ differed between the sucrose-associated and nonsucrose control levers (cue $\times$ lever interaction, $F_{(1,127)}=9.67$; $p<0.05)$. Only pressing on the sucrose lever was increased by presentation of the CS+ (two-way within-subject ANOVA with cue and drug as factors; main effect of cue, $F_{(1,63)}=20.65 ; p<$ $0.005)$. Pressing on the sucrose lever was reliably increased by presentation of the CS + for rats in the primary and nonlocalized groups, both after vehicle microinjection $\left(F_{(1,31)}=8.32 ; p<0.02\right)$ and after all doses of amphetamine ( $p<0.01$ for every dose), although the effect of the CS+ on pressing was much greater after amphetamine than after vehicle (described below).

\section{Control versus incentive effects}

Pressing on the control nonsucrose lever was not increased by presentation of the CS+ cue (two-way within-subject ANOVA with cue and drug as factors, $F_{(1,63)}=3.985 ; p=$ NS). Thus, the $\mathrm{CS}+$ sucrose cue selectively triggered increased lever pressing that 


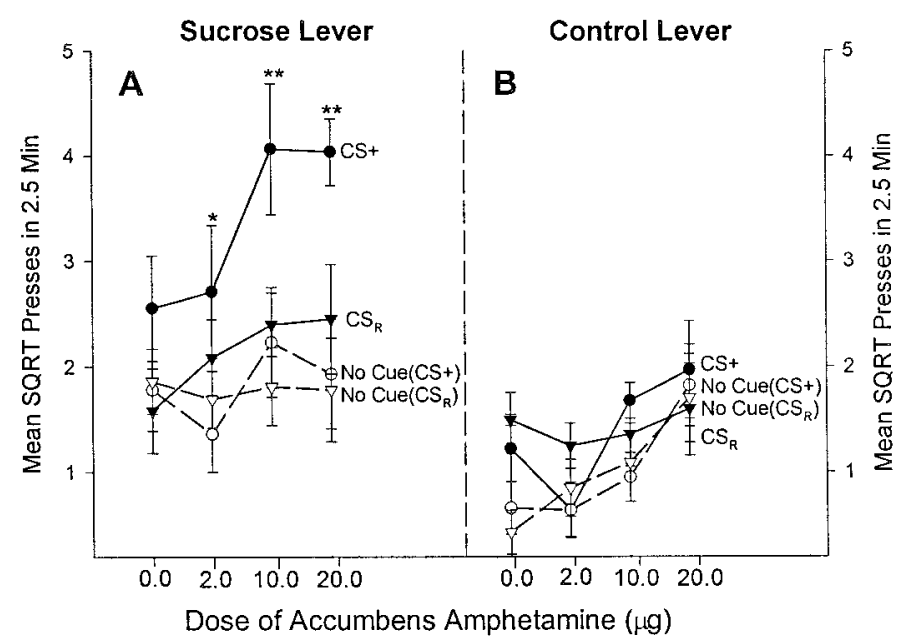

Figure 2. The effect of intra-accumbens amphetamine during lever pressing bouts in the presence and absence of the cue for both the CS + and $\mathrm{CS}_{\text {random }}$ groups. Filled circles represent mean \pm SEM squared root responses on each lever during presentations of the $\mathrm{CS}+$, and open circles represent responses during the periods immediately before each $\mathrm{CS}+$ presentation. Filled triangles depict responses during presentations of the $\mathrm{CS}_{\text {random }}$, and open triangles depict responses during the periods before each $\mathrm{CS}_{\text {rand }}$ presentation. Intra-accumbens amphetamine significantly increased the ability of the $\mathrm{CS}+$ to trigger enhanced responding on the sucrose lever (two-way ANOVA; ${ }^{*} p<0.01$ or $* * p<0.001$; Bonferroni) but not on the control lever. Amphetamine did not change the incentive impact of the $\mathrm{CS}_{\text {random }}$ control cue. In the absence of the $\mathrm{CS}+$ reward cue, amphetamine had only a minimal general motor effect on pressing of both levers.

was directed toward obtaining sucrose. Presentation of the CS nonconditioned cue slightly increased pressing on both levers (three-way within-subject ANOVA with cue, drug, and lever as factors; $\left.F_{(1,127)}=7.504 ; p<0.05\right)$, and there was no difference between the two levers in the magnitude of this enhancement (no significant cue $\times$ lever interaction; $F_{(1,127)}=0.050 ; p=\mathrm{NS}$ ). This indicates that the effect of the $\mathrm{CS}_{\text {random }}$ nonconditioned cue was to mildly enhance responding on both the sucrose and nonsucrose control levers indiscriminately, which is consistent with an increase in general activity.

\section{Effect of intra-accumbens amphetamine on lever pressing}

In the absence of cues, amphetamine microinjection produced an increase in pressing, especially on the control lever, and this effect occurred in both groups (one-way ANOVAs with drug as factor; $\mathrm{CS}+$ group, $F_{(3,31)}=4.698 ; p<0.05 ; \mathrm{CS}_{\text {random }}$ group, $F_{(3,31)}=$ $3.890 ; p<0.05$ ) (Fig. $2 B$ ). Although pressing on the sucrose lever in the absence of cues was not significantly enhanced by intraaccumbens amphetamine in either group (one-way within-subject ANOVAs with drug dose as factor; CS + group, $F_{(3,31)}=0.645, p=$ $\mathrm{NS}$; $\mathrm{CS}_{\text {random }}$ group, $\left.F_{(3,31)}=0.034 ; p=\mathrm{NS}\right)$, there was a trend of increased responding under the higher drug doses (Figs. $2 A, 3$ ) However, when the two levers were compared in terms of the percentage increase in pressing, amphetamine in the absence of the $\mathrm{CS}+$ cue produced a greater increase in pressing on the control lever than on the sucrose lever (one-way ANOVA with lever as factor; $F_{(1,15)}=14.347 ; p<0.001$ ) (Fig. $3 A$ ).

During the presentation of a reward cue, however, the effect of amphetamine was dramatically different from the effect in the absence of the cue. The CS + cue presence reversed the direction of amphetamine potentiation from the control lever to the sucrose lever and dramatically amplified the magnitude of response potentiation. Amphetamine specifically enhanced the ability of the CS+ to increase pressing on the sucrose lever in a dose-response manner (two-way within-subject ANOVA with drug and cue as factors; cue $\times$ drug interaction, $F_{(3,63)}=5.253 ; p<0.01$; post hoc comparisons for the effect of cue at each drug dose; vehicle, mean difference of $0.774, p=0.074 ; 2 \mu \mathrm{g}$, mean difference of $1.352, p<0.01$;
$10 \mu \mathrm{g}$, mean difference of 1.836, $p<0.001 ; 20 \mu \mathrm{g}$, mean difference of 2.104, $p<0.001$ ) (Fig. $2 A$ ). When reanalyzed in a separate one-way ANOVA, amphetamine doses again reliably enhanced responding on the sucrose lever during the $\mathrm{CS}+$ presentations $\left(F_{(3,31)}=3.228, p<0.05\right)$. In percentage terms, presentation of the $\mathrm{CS}+$ cue caused the amphetamine-induced increase in sucrose lever pressing to jump to nearly $400 \%$ from its $100 \%$ no-cue baseline (one-way within-subject ANOVA with cue as factor; $F_{(1,15)}$ $=6.000 ; p<0.05)$ (Fig. 3A). Intra-accumbens amphetamine did not, however, increase responding on the control lever during presentation of the CS+ sucrose cue (one-way ANOVA with drug as factor; $F_{(3,31)}=2.369, p=\mathrm{NS}$ ) (Fig. $2 B$ ). This suggests that the sucrose cue both redirected and further magnified the effect of intra-accumbens amphetamine, switching the enhancement of responding from the control lever to the sucrose lever in an incentive-specific manner. For the $\mathrm{CS}_{\text {random }}$ group, presentation of the $\mathrm{CS}_{\text {random }}$ control cue did not modify the effect of amphetamine on pressing of either the sucrose-associated lever (one-way ANOVA with drug as factor; $\left.F_{(3,31)}=1.084, p=\mathrm{NS}\right)$ or the nonsucrose control lever (one-way ANOVA with drug as factor; $\left.F_{(3,31)}=0.270 ; p=\mathrm{NS}\right)$.

\section{Nonlocalized CS control group}

For the nonlocalized CS control group, amphetamine microinjection similarly magnified the ability of an auditory $\mathrm{CS}+$ to elicit increased instrumental performance for sucrose. Presentation of the tone or clicker $\mathrm{CS}+$ triggered significantly greater pressing on the sucrose lever compared with pressing in the absence of the $\mathrm{CS}+$ or to pressing in the presence of the CS - (two-way ANOVAs with CS and drug as factors; main effect of CS+ presence, $F_{(1,31)}=$ 20.203, $p<0.01$; main effect of $C S+$ versus $C S-$ presence; $F_{(1,31)}$ $=10.157, p<0.05)$ (Fig. 4). Intra-accumbens amphetamine further potentiated the incentive impact of the auditory $\mathrm{CS}+$, selectively enhancing sucrose lever pressing in the presence the CS+ to $>200 \%$ of vehicle levels (post hoc comparison; $p<0.05$ ) (Fig. 4). In contrast, amphetamine had no effect on sucrose-associated lever pressing in the absence of the CSs (one-way ANOVAs with drug as factor; CS + absence, $F_{(1,15)}=0.109, p=\mathrm{NS}$; CS - absence, $F_{(1,15)}$ $=0.068, p=\mathrm{NS})$. Likewise, amphetamine had no effect on sucrose lever pressing in the presence of the CS- (two-way ANOVA with $\mathrm{CS}-$ presence and drug as factors; no effect of CS- presence, $F_{(1,31)}=0.814, p=\mathrm{NS}$; no effect of drug, $F_{(1,31)}=0.004, p=\mathrm{NS}$; no interaction, $\left.F_{(1,31)}=0.332, p=\mathrm{NS}\right)$. Finally, amphetamine did not alter responding on the nonsucrose control lever in the presence of the CS + (two-way ANOVA with drug and CS + presence as factors; no effect of drug, $F_{(1,31)}=1.806, p=$ NS; no effect of $\mathrm{CS}+$ presence, $F_{(1,31)}=0.803 ; p=\mathrm{NS}$; no interaction, $F_{(1,31)}=$ $0.803, p=\mathrm{NS})$. Thus, amphetamine microinjection in this nonlocalized CS control group specifically enhanced the ability of a nonlocalized reward cue to trigger increased seeking for sucrose reward.

\section{Does intra-accumbens amphetamine enhance both the conditioned incentive value and the hedonic impact of sucrose reward?}

One possible explanation of the increase in the motivational impact of the sucrose cue on instrumental performance is that amphetamine enhanced the hedonic impact that sucrose would have had and therefore increased the conditioned hedonic impact of the Pavlovian association of sucrose evoked by the cue. If intraaccumbens amphetamine enhanced the conditioned hedonic impact of a cue, then that enhancement of hedonia might have served to prime further responding, just as a free sucrose pellet could prime instrumental responding for sucrose (Toates, 1986). A very different interpretation, generated by the incentive salience hypothesis, is that the increased dopamine neurotransmission specifically boosted the attribution of incentive salience to the sucrose cue, allowing the cue to trigger "wanting" for sucrose but having no effect on "liking" for sucrose. In other words, according to the incentive salience hypothesis, intra-accumbens amphetamine may 

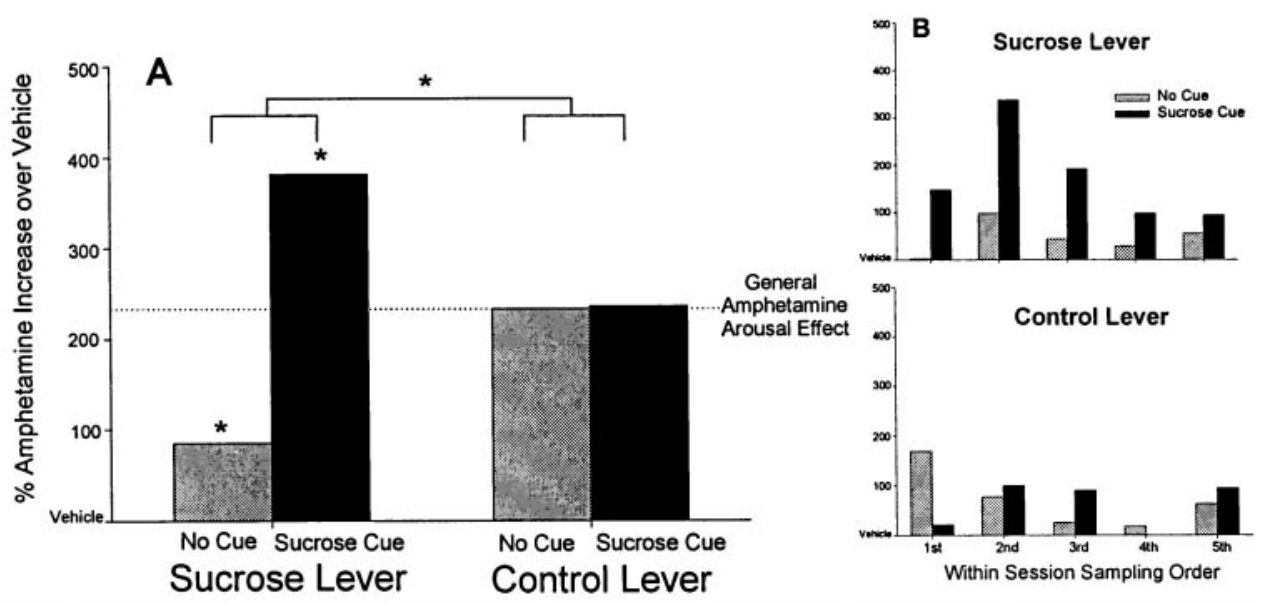

Figure 3. The percentage increase in lever pressing produced by amphetamine $(20 \mu \mathrm{g}, \mathrm{CS}+$ group $)$ compared with baseline pressing after vehicle microinjection. Amphetamine increased overall pressing on both the sucrose lever and the control lever $(A)$. In the absence of the cue, the percentage increase in pressing was greatest on the control lever. The presence of the CS + sucrose cue selectively enhanced pressing on the sucrose lever, producing a cue-triggered redirection of potentiation of behavior to obtain sucrose reward. $B$ shows the percentage increase in pressing produced by amphetamine during each successive CS + cue presentation and its paired no-cue period over the course of the test session. Amphetamine consistently enhanced pressing on the sucrose lever primarily during the CS + sucrose cue. The pattern of the effect by amphetamine on the control lever shows no relationship to the cue, in contrast, being sometimes greatest during the presence of the cue, sometimes during its absence, and sometimes producing little enhancement at all. Thus, pressing on both levers is sensitive to general arousal produced by amphetamine, but only pressing on the sucrose lever showed a percentage increase after amphetamine microinjection that was dramatically and reliably gated by the CS + reward cue.

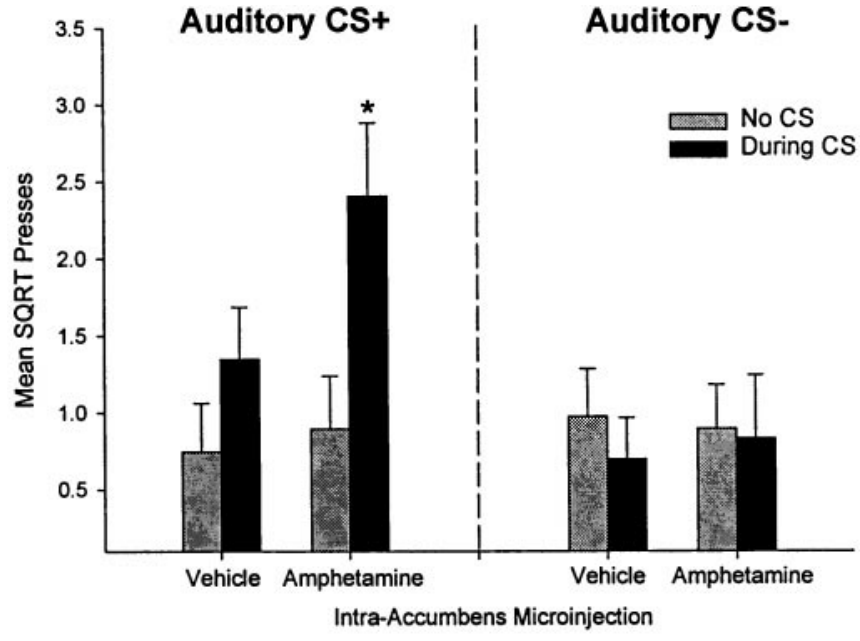

Figure 4. Nonlocalized CS + control group replicates the enhancement of the conditioned incentive effect of amphetamine. Lever pressing for sucrose under extinction conditions (after microinjection of amphetamine or vehicle) by the control group of rats that received Pavlovian training with auditory CS + and CS - (tone vs clicker). Amphetamine microinjection selectively enhanced sucrose lever pressing in the presence of the auditory $\mathrm{CS}+$ for sucrose. Pressing on the lever was not enhanced by amphetamine in the absence of the auditory CS + or in the presence of the auditory CS-.

potentiate incentive impact without necessarily potentiating hedonic impact.

\section{Experiment 2: taste reactivity testing}

The bittersweet sucrose-quinine solution evoked a mixture of hedonic and aversive reaction patterns, which allowed an assessment of whether the drug microinjections altered either hedonic impact or aversive impact (Fig. 5). Intra-accumbens amphetamine failed to enhance overall positive hedonic reactions compared with reactions elicited after vehicle microinjection. On the contrary, amphetamine produced a trend toward a dose-dependent suppression of sucrose-elicited hedonic reaction patterns $(p=0.098$; Friedman ANOVA), which became significant when the middle dose was excluded ( $p<0.05$; Friedman ANOVA). Furthermore, the pattern of affective reactions was distributed similarly over the various responses belonging to the positive hedonic category (e.g.,

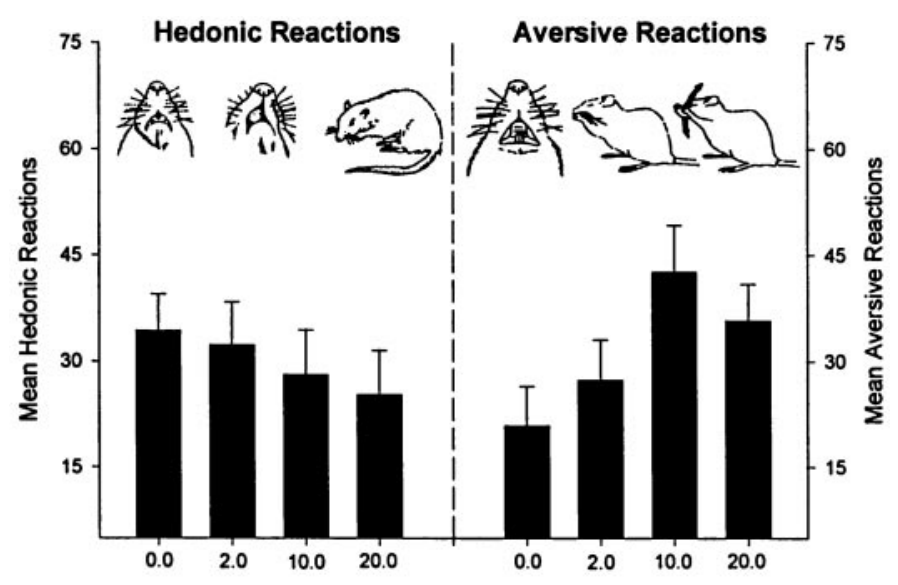

Dose of Accumbens Amphetamine ( $\mu \mathrm{g})$
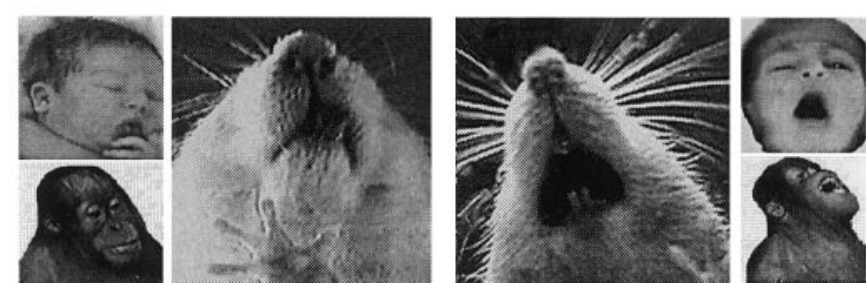

Figure 5. The effect of intra-accumbens amphetamine on affective reactions elicited by a bittersweet sucrose-quinine taste mixture. Error bars represent mean \pm SEM hedonic or aversive affective reactions. Overall hedonic reactions were mildly decreased by amphetamine $(p=0.098 ; p<$ 0.05 when the middle dose was excluded; Friedman ANOVA), whereas aversive taste reactions were conversely enhanced by amphetamine $(p<$ 0.05; Friedman ANOVA). These results are the opposite of what would be expected if amphetamine increased "liking" for the taste. Photos depict representative hedonic tongue protrusions and aversive gapes from an adult rat, infant human, and juvenile orangutan (modified from Berridge, 2000a).

rhythmic tongue protrusion, lateral tongue protrusion, and paw licking) (Fig. 6).

Overall aversive reactions to quinine were mildly changed in a reciprocal direction and were slightly enhanced after intra- 


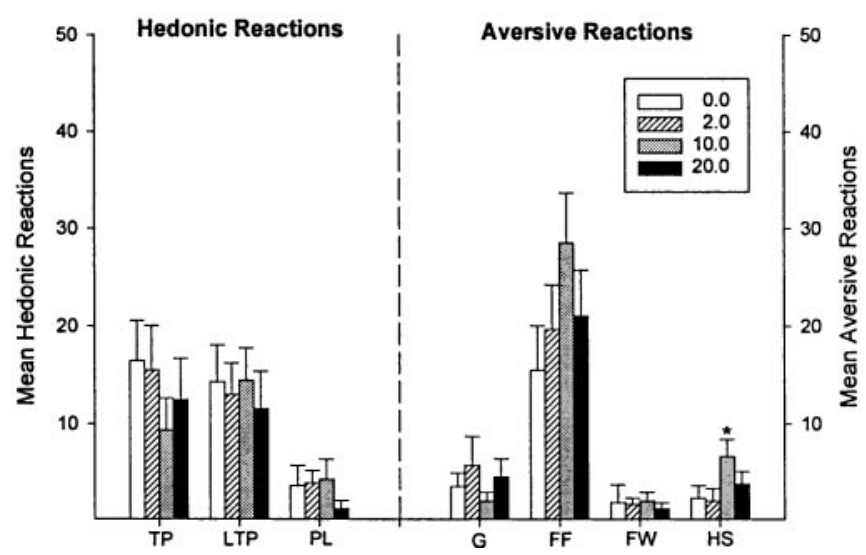

Figure 6. The pattern of hedonic and aversive affective reaction components elicited by the bittersweet sucrose-quinine taste is shown after microinjection of vehicle or amphetamine. Intra-accumbens amphetamine marginally decreased hedonic reaction components elicited by the sucrose taste and slightly increased aversive reaction components elicited by the quinine taste. That pattern is the opposite of what would be expected if amphetamine increased "liking" for the taste. Error bars represent mean 士 SEM total of each individual response component. TP, Tongue protrusions; $L T P$, lateral tongue protrusions; $P L$, paw licking; $F W$, face wiping; $F F$, forelimb flails; $G$, gapes; $H S$, head shakes.

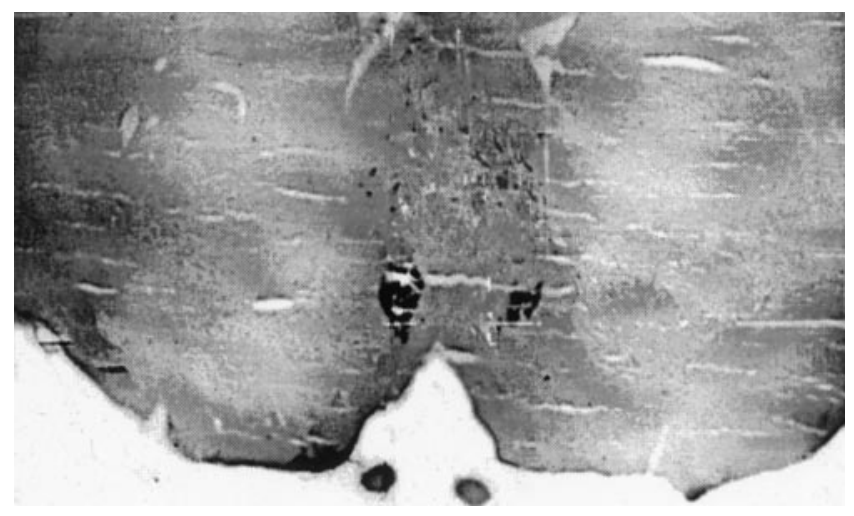

Figure 7. A photomicrograph of a representative brain slice.

accumbens amphetamine microinjection $(p<0.05$; Friedman ANOVA). When each behavioral component was analyzed separately, however, it was found that only aversive head shakes were significantly enhanced by amphetamine microinjection $(p<0.01$; Friedman ANOVA) (Fig. 6). Moreover, this effect was only significant at the middle dose $(10 \mu \mathrm{g})$, which might suggest a general motor enhancement effect on this response as an alternative explanation to a systematic enhancement of aversive taste reactivity. In any case, it was clear that amphetamine did not selectively enhance hedonic reaction patterns as would be expected if amphetamine increased the hedonic impact of sucrose. The pattern of data instead indicates that amphetamine either had little effect at all on hedonic impact or else shifted palatability slightly in the negative direction.

\section{Histology}

A photomicrograph of a representative bilateral microinjection site is shown in Figure 7. All microinjection placements are shown in Figure 8. Microinjection sites were found to be primarily restricted to the caudomedial shell of the nucleus accumbens in rats from both experiments.

These results indicate that microinjection of amphetamine into the nucleus accumbens failed to enhance the hedonic palatability of the sucrose-quinine mixture. There was no evidence that intraaccumbens amphetamine increased the hedonic impact or "liking" of the sucrose element in the bittersweet taste. Similarly, there was

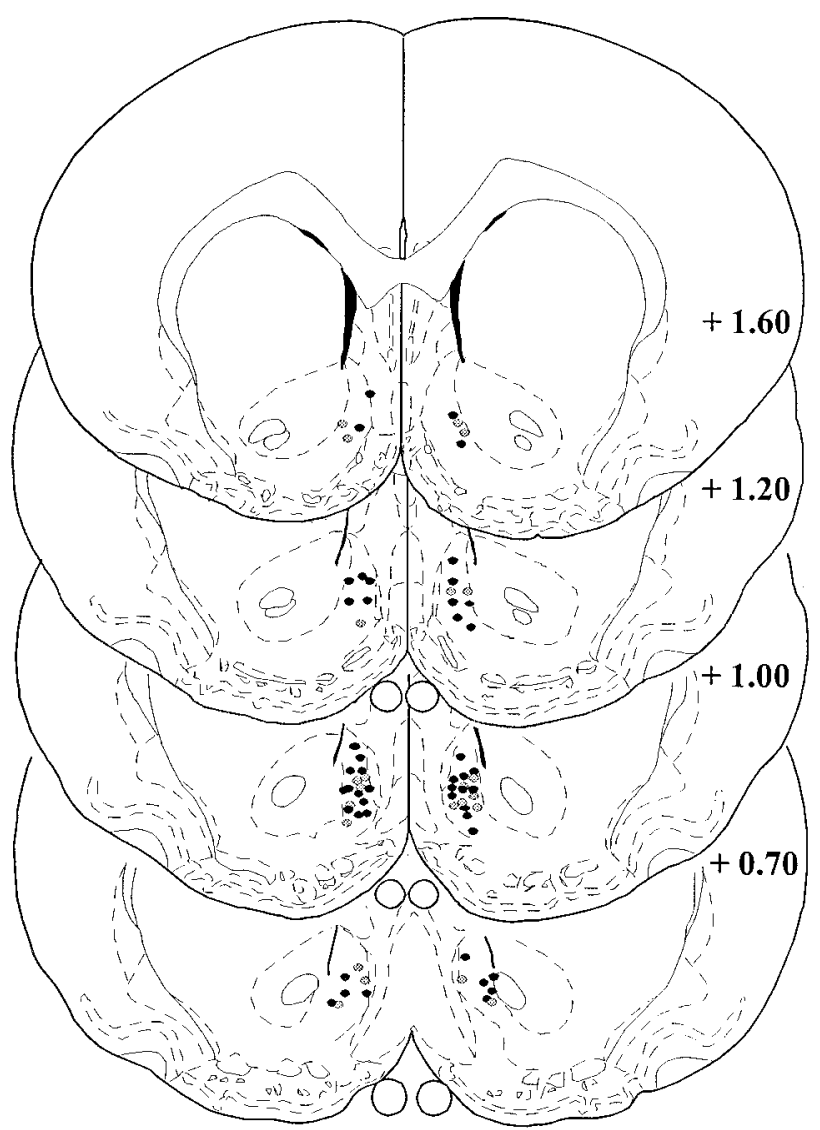

Figure 8. A schematic representation of the microinjection placement sites in the shell of the nucleus accumbens. Black circles represent subjects from experiment 1, and gray circles represent subjects from experiment 2 . The drawing was adapted from Paxinos and Watson (1986), and the numbers denote distance in millimeters from bregma.

no evidence that amphetamine reduced the aversive impact of the quinine element. The only significant reaction changes were in the opposite direction, because amphetamine produced a mild enhancement of aversive taste reactivity, but there was not a consistent increase in the overall pattern of aversive reactions. In summary, there was no enhancement of hedonic palatability produced by amphetamine microinjection.

It is important to note that the failure of amphetamine to enhance taste hedonic impact in this experiment occurred across a range of drug doses that have been reported previously to have positive effects on incentive motivation and reward. The lowest dose of intra-accumbens amphetamine $(2 \mu \mathrm{g})$ has been reported to enhance feeding (Sills and Vaccarino, 1996), the middle dose (10 $\mu \mathrm{g})$ has been shown to produce a conditioned place preference (Carr and White, 1986), and the highest dose (20 $\mu \mathrm{g})$ has been reported to enhance responding in the conditioned reinforcement paradigm (Kelley and Delfs, 1991). Moreover, all three of these amphetamine doses enhanced sucrose cue-elicited instrumental responding for sucrose in experiment 1 . Thus, the inability of intra-accumbens amphetamine to enhance hedonic reactions to taste cannot be dismissed on the grounds that these drug doses were inappropriate; they have been shown repeatedly to produce rewarding effects, to enhance feeding, and to potentiate the incentive motivation for food reward in our pure conditioned incentive experiment. In other words, these doses of amphetamine have reward- or motivation-enhancing effects relevant to food incentives, but our observations indicate those reward-relevant effects do not extend to increasing the hedonic impact of a taste.

\section{DISCUSSION}

These experiments demonstrate that amphetamine microinjection into the accumbens shell potentiates the ability of a Pavlovian 
sucrose cue to trigger increases in instrumental responding for sucrose reward, even in the absence of either primary response reinforcement by actual sucrose reward or of secondary response reinforcement by the sucrose cue. Instrumental responding was increased up to $400 \%$ by intra-accumbens amphetamine during presentation of the $\mathrm{CS}+$ sucrose cue but not during presentation of the control $\mathrm{CS}_{\text {random }}$ or $\mathrm{CS}-$ cues. In the absence of any cue, amphetamine microinjection produced a greater percentage increase in pressing on the control lever rather than on the sucrose lever (although the absolute rate of pressing on the control lever was always low). However, during the $\mathrm{CS}+$ sucrose cue presence, that relation was reversed, and amphetamine microinjection produced a much greater percentage increase in pressing on the sucrose lever than on the control lever. Thus, the presence of the sucrose cue redirected the amphetamine enhancement of pressing away from the control lever and toward the sucrose lever and greatly multiplied its effect. Therefore, this amphetamine-mediated enhancement of responding reflects a specific increase in the cuetriggered effort to obtain sucrose reward.

\section{Sensorimotor arousal versus incentive value of food}

In the absence of cues, the only effect of amphetamine microinjection was to slightly increase responding on both levers, especially the nonsucrose control lever. This suggests that amphetamine microinjection induced a modest increase in sensorimotor responsiveness, which is consistent with the hypothesis that mesolimbic dopamine is involved in sensorimotor and integrative functions involved in generating complex responses (Salamone, 1986; Salamone et al., 1997). However, the magnitude of this general sensorimotor arousal increase was always small. In contrast, the druginduced enhancement of sucrose-specific responding observed during the sucrose cue was much larger (e.g., 400\%).

\section{Reversible motivational state, not an enduring cognitive expectation of food}

Intra-accumbens amphetamine enhanced the ability of the sucrose cue to trigger pressing on the sucrose lever, but responding dropped back to the vehicle baseline rate once the cue was removed. The reversibility of the cue-triggered increase in responding produced by amphetamine indicates that it required actual perception of the presence of the sucrose cue. This is consistent with the hypothesis that dopamine activation increases the incentive salience of a perceived Pavlovian reward cue, causing the cue to evoke a temporary motivational state of "wanting" for the reward (Berridge and Robinson, 1998; Berridge, 2000b).

\section{Not an enhancement of hedonic impact}

Intra-accumbens amphetamine failed to enhance the hedonic impact of sucrose reward, although it enhanced the conditioned incentive motivation for sucrose reward. Hedonic taste reactivity patterns elicited by sucrose-quinine were slightly shifted toward aversion, if changed at all, after amphetamine microinjection. That pattern of affective reactions is the opposite of what would be expected if amphetamine enhanced the hedonic impact of sucrose reward. The failure of amphetamine microinjection to enhance positive hedonic taste reactivity patterns is especially striking because morphine microinjection at a similar accumbens site does enhance positive hedonic reactions elicited by sucrose (Peciña and Berridge, 2000). Thus, opioid receptor activation in the accumbens shell increases both "liking" and "wanting" for food, whereas dopamine receptor activation fails to enhance sucrose "liking," although it clearly enhanced conditioned sucrose wanting in this study.

The inability of intra-accumbens amphetamine to enhance taste hedonic impact is consistent with the results of previous taste reactivity studies that have manipulated dopamine systems. Neither systemic dopamine antagonists (Treit and Berridge, 1990; Peciña et al., 1997) nor depletion of up to $99 \%$ of mesolimbic and nigrostriatal dopamine by 6-hydroxydopamine lesions suppress "liking" for a food reward as assessed by hedonic taste reactivity (Berridge et al., 1989; Berridge and Robinson, 1998).

\section{Not an enhancement of conditioned reinforcement}

It has often been posited that Pavlovian reward cues act primarily as secondary reinforcers that strengthen preceding responses. However, to act as a conditioned reinforcer, the cue must be contingently presented after reinforced responses. Our results cannot be explained by an amphetamine-induced enhancement of response reinforcement because the conditioned incentive paradigm precluded all reinforcement contingencies during the test.

Just as amphetamine had no chance to enhance the primary reinforcement value of sucrose in experiment 1 (because rats were tested only in extinction, when sucrose was no longer earned by pressing on the sucrose lever), there was also no opportunity for intra-accumbens amphetamine to enhance cue-mediated secondary reinforcement (because there was no contingency between lever pressing and cue presentation). We never presented the cue as a contingent reinforcer for preceding responses but instead presented it freely every few minutes to elicit further subsequent responses. Our rats in experiment 1 were working for real sucrose (albeit in extinction) and not for the cue, which occurred independent of whether the rats pressed or not. Thus, our results cannot be explained by an amphetamine-induced enhancement of either primary or secondary reinforcement, although intra-accumbens amphetamine is known to enhance secondary reinforcement when response reinforcement contingencies exist (Taylor and Robbins, 1984; Robbins et al., 1989; Cador et al., 1991; Kelley and Delfs, 1991; Everitt and Robbins, 1992).

It could be argued that amphetamine microinjection might have inadvertently enhanced the secondary reinforcement value of stimuli other than the sucrose cue, such as the sucrose lever or the act of pressing it. However, if that had happened, then pressing on the sucrose lever should have been elevated even in the absence of the $\mathrm{CS}+$, because the lever and other stimuli were always present. However, pressing on the sucrose lever was only slightly enhanced by amphetamine in the absence of the sucrose cue.

Together, these results show that intra-accumbens amphetamine increased the pure conditioned incentive impact of the reward cue. That is, amphetamine magnified the ability of a CS + cue to trigger instrumental behavior for its associated sucrose reward by acting in a purely conditioned incentive manner rather than through primary or secondary response reinforcement.

\section{Enhancement of incentive salience attributed to CS+}

Our results are consistent with the hypothesis that intra-accumbens amphetamine increased the sucrose-related incentive salience attributed to the CS+ sucrose cue, causing it to trigger increased "wanting" for sucrose but not "liking" for sucrose. According to the incentive salience hypothesis, the attribution of incentive salience to a sucrose cue would boost behavior in two ways. First, by making the cue light located on the sucrose lever more attractive, the cue would become a more potent elicitor of approach responses (in a manner similar to autoshaping) (Brown and Jenkins, 1968; Beagley and Holley, 1977; Rescorla and Cunningham, 1979; Davey et al., 1984; Toates, 1986, 1994; Oscos et al., 1988; Berridge, 1996; Tomie, 1996). Second, amphetamine would have increased cue-triggered "wanting" for sucrose itself by increasing the incentive salience of the cue-triggered neural representation of sucrose reward (Robinson and Berridge, 1993; Berridge and Robinson, 1998; Berridge, 2000b).

Our conclusion that mesolimbic dopamine activation modulates the incentive salience of a Pavlovian sucrose cue is consistent with recent findings by Dickinson and colleagues who have demonstrated a converse suppression of conditioned incentive salience by dopamine antagonists. Dickinson et al. (2000) found that systemic pimozide or $\alpha$-flupenthixol specifically suppressed the ability of a Pavlovian food CS + to trigger instrumental performance for food under extinction conditions. In contrast, baseline performance (more related to expectations of hedonic value based on previous experience) was not affected. Regarding theories of dopamine function, Dickinson et al. (2000) concluded that their "results are most readily integrated with that advanced by Berridge and Rob- 
inson (Robinson and Berridge, 1993; Berridge and Robinson, 1998). According to this theory, food rewards activate two distinctive but interacting systems: a DA-dependent incentive salience system and a DA-independent hedonic system" (Dickinson et al., 2000). Thus, the DA-dependent system seems to be responsible specifically for enhancing the conditioned incentive impact of reward cues (Dickinson et al., 2000) (for discussion, see Berridge, 2000b).

Our conclusion is also consistent with the Incentive-Sensitization theory of addiction (Robinson and Berridge, 1993, 2000), which posits that neural sensitization increases the conditioned incentive salience of drug-associated stimuli for addicts, causing them to "want" drugs excessively. Indeed, preliminary observations from our laboratory indicate that rats sensitized by previous psychostimulant drug administration exhibit excessive cue-triggered reward-seeking behavior in this pure conditioned incentive paradigm (Wyvell and Berridge, 2000). These results are compatible with other evidence that sensitization increases the conditioned incentive value of reward-related stimuli (Harmer and Phillips, 1998; De Vries et al., 1999; Fiorino and Phillips, 1999; Taylor and Horger, 1999).

\section{Accumbens site of action}

The accumbens shell was targeted here because a special role for the shell has been implicated in dopamine activation by palatable food or food cues (Park and Carr, 1998; Bassareo and Di Chiara, 1999) and in the elicitation of feeding behavior (Swanson et al., 1997; Basso and Kelley, 1999; Peciña and Berridge, 2000). Shell lesions abolish the ability of intra-accumbens amphetamine to potentiate responding for food conditioned reinforcers, whereas lesions of the accumbens core disrupt other learning-related aspects of conditioned reinforcement (Parkinson et al., 1999). In short, the accumbens shell plays a special role in food reward, but the core may also be important.

Although our microinjections were placed into the accumbens shell, the spread of the drug microinjection was unknown. Future studies will be needed to verify whether or not the accumbens shell specifically mediates the enhancement of pure conditioned incentive motivation demonstrated here.

\section{Conclusion}

Intra-accumbens amphetamine increased the ability of a sucrose cue to spur performance for sucrose reward, even under extinction conditions that precluded mediation by primary or secondary response reinforcement. The enhancement of reward "wanting" was directly triggered by the perception of the CS + sucrose cue. Intra-accumbens amphetamine did not enhance "liking" of sucrose as measured by the hedonic taste reactivity paradigm, although the same doses enhanced "wanting" for sucrose. Together, these results support the hypothesis that accumbens amphetamine specifically enhances the attribution of incentive salience to Pavlovian reward cues. In other words, mesoaccumbens dopamine neurotransmission mediates the ability of reward cues to trigger "wanting" for their associated rewards.

\section{REFERENCES}

Ahn S, Phillips AG (1999) Dopaminergic correlates of sensory-specific satiety in the medial prefrontal cortex and nucleus accumbens of the rat. J Neurosci 19:RC29.

Balleine B (1994) Asymmetrical interactions between thirst and hunger in Pavlovian-instrumental transfer. Q J Exp Psychol 47B:211-231.

Bassareo V, Di Chiara G (1999) Differential responsiveness of dopamine transmission to food-stimuli in nucleus accumbens shell/core compartments. Neuroscience 89:637-641.

Basso AM, Kelley AE (1999) Feeding induced by GABA(A) receptor stimulation within the nucleus accumbens shell: regional mapping and characterization of macronutrient and taste preference. Behav Neurosci 113:324-336.

Beagley WK, Holley TL (1977) Hypothalamic stimulation facilitates contralateral visual control of a learned response. Science 196:321-323.

Beauchamp GK, Bertino M, Burke D, Engelman K (1990) Experimental sodium depletion and salt taste in normal human volunteers. Am J Clin Nutr 51:881-889.
Berridge KC (1991) Modulation of taste affect by hunger, caloric satiety, and sensory-specific satiety in the rat. Appetite 16:103-120.

Berridge KC (1996) Food reward: brain substrates of "wanting" and "liking." Neurosci Biobehav Rev 20:1-25.

Berridge KC (2000a) Taste reactivity: measuring hedonic impact in human infants and animals. Neurosci Biobehav Rev 24:173-198.

Berridge KC (2000b) Reward learning: reinforcement, incentives, and expectations. In: The psychology of learning and motivation, Vol 40 (Medin D, ed). New York: Academic, in press.

Berridge KC, Peciña S (1995) Benzodiazepines, appetite, and taste palatability. Neurosci Biobehav Rev 19:121-131.

Berridge KC, Robinson TE (1998) What is the role of dopamine in reward: hedonic impact, reward learning, or incentive salience? Brain Res Rev 28:309-369.

Berridge KC, Schulkin J (1989) Palatability shift of a salt-associated incentive during sodium depletion. Q J Exp Psychol 41B:121-138.

Berridge KC, Valenstein ES (1991) What psychological process mediates feeding evoked by electrical stimulation of the lateral hypothalamus? Behav Neurosci 105:3-14.

Berridge KC, Flynn FW, Schulkin J, Grill HJ (1984) Sodium depletion enhances salt palatability in rats. Behav Neurosci 98:652-660.

Berridge KC, Venier IL, Robinson TE (1989) Taste-reactivity analysis of 6-hydroxydopamine-induced aphagia: implications for arousal and anhedonia hypotheses of dopamine function. Behav Neurosci 103:36-45.

Blackburn JR, Phillips AG, Jakubovic HC, Fibiger HC (1989) Dopamine and preparatory behavior. II. A neurochemical analysis. Behav Neurosci 103:15-23.

Breslin PA, Davidson TL, Grill HJ (1990) Conditioned reversal of reactions to normally avoided tastes. Physiol Behav 47:535-538.

Brown PL, Jenkins HM (1968) Auto-shaping of the pigeon's key-peck. J Exp Anal Behav 11:1-8.

Burns LH, Robbins TW, Everitt BJ (1993) Differential effects of excitotoxic lesions of the basolateral amygdala, ventral subiculum and medial prefrontal cortex on responding with conditioned reinforcement and locomotor activity potentiated by intra-accumbens infusions of D-amphetamine. Behav Brain Res 55:167-183.

Cabanac M (1979) Sensory pleasure. Q Rev Biol 54:1-29.

Cador M, Robbins TW, Everitt BJ, Simon H, Le Moal M, Stinus L (1991) Limbic-striatal interactions in reward-related processes: modulation by the dopaminergic system. In: The mesolimbic dopamine system: from motivation to action (Willner P, Scheel-Kruger J, eds), pp 449-474. New York: Wiley.

Carr GD, White NM (1986) Anatomical dissociation of amphetamine's rewarding and aversive effects: an intracranial microinjection study. Psychopharmacology 89:340-346.

Davey GC, Cleland GG, Oakley DA, Jacobs JL (1984) The effect of early feeding experience on signal-directed response topography in the rat. Physiol Behav 32:11-15.

Delamater AR, LoLordo VM, Berridge KC (1986) Control of fluid palatability by exteroceptive Pavlovian signals. J Exp Psychol Anim Behav Process 12:143-152.

De Vries TJ, Schoffelmeer ANM, Binnekade R, Vanderschuren L (1999) Dopaminergic mechanisms mediating the incentive to seek cocaine and heroin following long-term withdrawal of IV drug self-administration. Psychopharmacology 143:254-260.

Di Chiara G (1998) A motivational learning hypothesis of the role of mesolimbic dopamine in compulsive drug use. J Psychopharmacol 12:54-67.

Di Ciano P, Blaha CD, Phillips AG (1998a) The relation between oxidation currents in the nucleus accumbens and conditioned increases in motor activity in rats following repeated administration of D-amphetamine or cocaine. Eur J Neurosci 10:1113-1120.

Di Ciano P, Blaha CD, Phillips AG (1998b) Conditioned changes in oxidation currents in the nucleus accumbens of rats by stimuli paired with self-administration or yoked administration of D-amphetamine. Eur J Neurosci 10:1121-1127.

Dickinson A, Balleine B (1994) Motivational control of goal-directed action. Anim Learn Behav 22:1-18.

Dickinson A, Dawson GR (1987) Pavlovian processes in the motivational control of instrumental performance. Q J Exp Psychol B 39:201-213.

Dickinson A, Smith J, Mirenowicz J (2000) Dissociation of Pavlovian and instrumental incentive learning under dopamine antagonists. Behav Neurosci 114:468-483.

Doyle TG, Berridge KC, Gosnell BA (1993) Morphine enhances hedonic taste palatability in rats. Pharmacol Biochem Behav 46:745-749.

Everitt BJ, Robbins TW (1992) Amygdala-ventral striatal interactions and reward-related processes. In: The amygdala: neurobiological aspects of emotion, memory, and mental dysfunction (Appleton JP, ed), pp 401429. New York: Wiley-Liss.

Everitt BJ, Cardinal RN, Hall J, Parkinson JA, Robbins TW (2000) Differential involvement of amygdala subsystems in appetitive conditioning and drug addiction. In: The amygdala: a functional analysis (Appleton JP, ed). New York: Wiley-Liss, in press.

Fiorino DF, Phillips AG (1999) Facilitation of sexual behavior in male rats following D-amphetamine-induced behavioral sensitization. Psychopharmacology 142:200-208.

Gardner EL, Lowinson JH (1993) Drug craving and positive-negative 
hedonic brain substrates activated by addicting drugs. Semin Neurosci 5:359-368.

Glantz SA (1997) Primer of biostatistics, pp 313-314. New York: McGraw-Hill.

Grill HJ, Berridge KC (1985) Taste reactivity as a measure of the neural control of palatability. In: Progress in psychobiology and physiological psychology (Sprague JM, Epstein AN, eds), pp 1-61. Orlando, FL: Academic.

Grill HJ, Norgren R (1978) The taste reactivity test. I. Mimetic responses to gustatory stimuli in neurologically normal rats. Brain Res 143:263-279.

Harmer CJ, Phillips GD (1998) Enhanced appetitive conditioning following repeated pretreatment with D-amphetamine. Behav Pharmacol 9:299-308.

Hernandez L, Hoebel BG (1988) Food reward and cocaine increase extracellular dopamine in the nucleus accumbens as measured by dialysis. Life Sci 42:1705-1712.

Kelley AE, Delfs JM (1991) Dopamine and conditioned reinforcement. I. Differential effects of amphetamine microinjections into striatal subregions. Psychopharmacology 103:187-196.

Laeng B, Berridge KC, Butter CM (1993) Pleasantness of a sweet taste during hunger and satiety: effects of gender and "sweet tooth." Appetite $21: 247-254$.

Mark GP, Smith SE, Rada PV, Hoebel BG (1994) An appetitively conditioned taste elicits a preferential increase in mesolimbic dopamine release. Pharmacol Biochem Behav 48:651-660.

McFarland K, Ettenberg A (1998) Haloperidol does not affect motivational processes in an operant runway model of food-seeking behavior. Behav Neurosci 112:630-635.

Oscos A, Martinez Jr JL, McGaugh JL (1988) Effects of post-training D-amphetamine on acquisition of an appetitive autoshaped lever press response in rats. Psychopharmacology 95:132-134.

Park TH, Carr KD (1998) Neuroanatomical patterns of fos-like immunoreactivity induced by a palatable meal and meal-paired environment in saline- and naltrexone-treated rats. Brain Res 805:169-180.

Parkinson JA, Olmstead MC, Burns LH, Robbins TW, Everitt BJ (1999) Dissociation in effects of lesions of the nucleus accumbens core and shell on appetitive Pavlovian approach behavior and the potentiation of conditioned reinforcement and locomotor activity by D-amphetamine. J Neurosci 19:2401-2411.

Paxinos G, Watson C (1986) The rat brain in stereotaxic coordinates, Ed 2. Sydney: Academic.

Peciña S, Berridge KC (1996) Brainstem mediates diazepam enhancement of palatability and feeding: microinjections into fourth versus lateral ventricle. Brain Res 727:22-30.

Peciña S, Berridge KC (2000) Opioid site in nucleus accumbens shell for food intake and hedonic impact: mapping by microinjection Fos plumes. Brain Res 863:71-86.

Peciña S, Berridge KC, Parker LA (1997) Pimozide does not shift palatability: separation of anhedonia from sensorimotor effects. Pharmacol Biochem Behav 58:801-811.

Phillips AG, Atkinson LJ, Blackburn JR, Blaha CD (1993) Increased extracellular dopamine in the nucleus accumbens of the rat elicited by a conditioned stimulus for food: an electrochemical study. Can J Physiol Pharmacol 71:387-393.

Phillips GD, Robbins TW, Everitt BJ (1994) Mesoaccumbens dopamineopiate interactions in the control over behaviour by a conditioned reinforcer. Psychopharmacology 114:345-359.

Rescorla RA (1967) Pavlovian conditioning and its proper control procedures. Psychol Rev 74:71-80.

Rescorla RA, Cunningham CL (1979) Spatial contiguity facilitates Pav- lovian second-order conditioning. J Exp Psychol Anim Behav Process 5:152-161.

Rescorla RA, Solomon RL (1967) Two-process learning theory: Relationship between Pavlovian and instrumental learning. Psychol Rev $74: 151-182$.

Richardson NR, Gratton A (1996) Behavior-relevant changes in nucleus accumbens dopamine transmission elicited by food reinforcement: an electrochemical study in rat. J Neurosci 16:8160-8169.

Robinson TE, Berridge KC (1993) The neural basis of drug craving: an incentive-sensitization theory of addiction. Brain Res Rev 18:247-291.

Robinson TE, Berridge KC (2000) The psychology and neurobiology of addiction: an incentive sensitization view. Addiction 95:S91-S117.

Robbins TW, Cador M, Taylor JR, Everitt BJ (1989) Limbic-striatal interactions in reward-related processes. Neurosci Biobehav Rev 13:155-162.

Salamone JD (1986) Different effects of haloperidol and extinction in instrumental behaviours. Psychopharmacology 88:18-23.

Salamone JD, Cousins MS, Snyder BJ (1997) Behavioral functions of nucleus accumbens dopamine: empirical and conceptual problems with the anhedonia hypothesis. Neurosci Biobehav Rev 21:341-359.

Schultz W, Tremblay L, Hollerman JR (1998) Reward prediction in primate basal ganglia and frontal cortex. Neuropharmacology 37:421-429.

Sills TL, Vaccarino FJ (1996) Individual differences in sugar consumption following systemic or intraaccumbens administration of low doses of amphetamine in nondeprived rats. Pharmacol Biochem Behav 54:665-670.

Sokolowski JD, Salamone JD (1998) The role of accumbens dopamine in lever pressing and response allocation: Effects of 6-OHDA injected into core and dorsomedial shell. Pharmacol Biochem Behav 59:557-566.

Swanson CJ, Heath TR, Stratford TR, Kelley AE (1997) Differential behavioral responses to dopaminergic stimulation of nucleus accumbens subregions in the rat. Pharmacol Biochem Behav 58:933-945.

Taylor JR, Horger BA (1999) Enhanced responding for conditioned reward produced by intra-accumbens amphetamine is potentiated after cocaine sensitization. Psychopharmacology 142:31-40.

Taylor JR, Robbins TW (1984) Enhanced behavioral control by conditioned reinforcers following microinjections of D-amphetamine into the nucleus accumbens. Psychopharmacology 84:405-412.

Toates F (1986) Motivational systems. Cambridge, UK: Cambridge UP.

Toates F (1994) Comparing motivational systems: an incentive motivational perspective. In: Appetite: neural and biobehavioural bases (Legg CR, Booth DA, eds), pp 305-327. New York: Oxford UP.

Tomie A (1996) Locating reward cue at response manipulandum (CAM) induces symptoms of drug abuse. Neurosci Biobehav Rev 20:505-535.

Treit D, Berridge KC (1990) A comparison of benzodiazepine, serotonin, and dopamine agents in the taste-reactivity paradigm. Pharmacol Biochem Behav 37:451-456.

Volkow ND, Wang GJ, Fowler JS, Logan J, Gatley SJ, Wong C, Hitzemann R, Pappas NR (1999) Reinforcing effects of psychostimulants in humans are associated with increases in brain dopamine and occupancy of $\mathrm{D}(2)$ receptors. J Pharmacol Exp Ther 291:409-415.

Wise RA (1985) The anhedonia hypothesis: mark III. Behav Brain Sci 8:178-186.

Wise RA, Spindler J, deWit H, Gerberg GJ (1978) Neuroleptic-induced "anhedonia" in rats: pimozide blocks reward quality of food. Science 201:262-264.

Wyvell CL, Berridge KC (2000) Intra-accumbens amphetamine enhances the conditioned incentive salience of sucrose reward. Soc Neurosci Abstr, in press. 